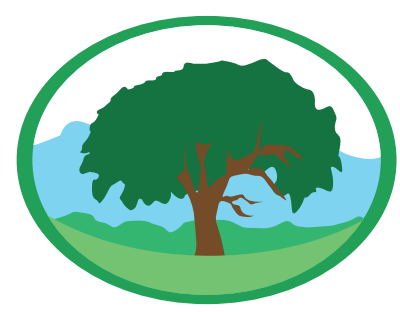

\title{
Tendências de séries de precipitação total mensal para localidades do Rio Grande do Sul
}

SILVA, G.M. ${ }^{1}$, TEIXEIRA-GANDRA, C.F.A. ${ }^{2}$, DAMÉ, R.C.F. ${ }^{2}$, KLUMB, G.B. ${ }^{3}$, VEBER, P.M. ${ }^{3}$

${ }^{1}$ Geógrafa, Mestranda no Programa de Pós-Graduação em Manejo e Conservação do Solo e da Água, UFPel/Pelotas - RS, Fone: (53) 3921-1433, giselesilvalacavg.ifsul.edu.br

${ }^{2}$ Eng ${ }^{\circ}$. Agrícola, Prof. Associada, Centro de Engenharias, UFPel/Pelotas - RS, cfteixeidig.com.br, ritah2olahotmail.com

${ }^{3}$ Acadêmico do Curso de Eng ${ }^{\circ}$. Agrícola, Centro de Engenharias, UFPel/Pelotas - RS,gustavo19klumbahotmail.com, patrick. veberahotmail.com

Palavras-chave: alteração

climática, análise de independência, teste não paramétrico

\begin{abstract}
Resumo
Evidências científicas sobre as alterações climáticas em nível mundial vêm despertando interesses crescentes na comunidade científica em geral. A importância na detecção das referidas alterações reside no fato de que uma vez detectada é possível o levantamento da situação, objetivando dar um panorama do comportamento hídrico da região. 0 presente trabalho teve como objetivo identificar a presença ou ausência de tendência nas séries de precipitação total mensal em 40 estações pluviométricas, localizadas nas sete mesorregiões do estado do Rio Grande do Sul, totalizando para cada estação, 42 anos (19612002), obtidas do banco de dados da Agência Nacional de Águas. Inicialmente foi realizada a verificação da independência das séries temporais por meio do teste de autocorrelação serial e após a detecção da existência de tendência monotônica e pontos de mudança, a partir dos testes não paramétricos de Mann-Kendall e Mann-Whitney. Das 40 estações analisadas, apenas 6 foram consideradas independentes e destas a localidade de Pelotas apresentou alteração de tendência quando da utilização do teste não paramétrico de Mann-Whitney, que utiliza séries subdivididas, comparativamente ao teste de Mann-Kendall, que utiliza a série completa, evidenciando que o mesmo é menos robusto.
\end{abstract}

\section{Analysis of precipitation series of total monthly trend for Rio Grande do Sul}

Key words: climate change, independent analysis, non parametric test

\begin{abstract}
Scientific evidence on climate change at the global level have attracted growing interest in the scientific community. The importance of detecting these changes lies in the fact that once detected can survey the situation, aiming to give an overview of the water behavior in the region. This work had the primary goal, identify the presence or absence of trend in monthly precipitation series in 40 rain stations located in the seven mesoregions of Rio Grande do Sul state, totaling 42 years (1961-2002), obtained of the Agência Nacional de Águas. Initially it was performed to verify the independence of the time series through the serial autocorrelation test and after the detection of the existence of monotonic trend and change points from the non parametric Mann-Kendall and Mann-Whitney. Of the 40 stations analyzed, only six were found to be independent and these the town of Pelotas showed a change trend when using the non parametric Mann-Whitney test, which uses split series, compared with the Mann-Kendall test, which uses the full range.
\end{abstract}




\section{INTRODUC̣ÃO}

Estudos sobre a climatologia de um local ou região tratam de caracterizar o estado médio da atmosfera em um determinado espaço de tempo, com foco nas mudanças antropogênicas, ou seja, a influência das atividades humanas sobre o meio ambiente. Tais mudanças têm sido alvo de vários estudos sobre aquecimento global, mudanças climáticas e cenários futuros (ALCÂNTARA et al., 2012; VENKATARAMAN et al., 2012). Ribeiro et al. (2014) definem a tendência climática como uma alteração suave do clima, com acréscimo ou decréscimo nos valores médios das variáveis analisadas, no período de registro. Assim, os impactos causados pelas alteraçóes climáticas e açôes antropogênicas na bacia hidrográfica em estudo podem ocasionar erros nos projetos do sistema hidráulico do local (TEIXEIRA et al., 2011).

Silva et al. (2010) analisando séries temporais de precipitação de todo o Brasil para o período de 1961 a 2008 encontraram padróes diferenciados e muito regionalizados nas tendências de aumento $e$ diminuição das precipitaçôes. Em termos anuais, a faixa leste da região Sul do Brasil destaca-se como área com tendências positivas significativas, desde o centro do Rio Grande do Sul até o Paraná. Segundo Minuzzi e Caramori (2011), desde meados do século XX, o estado do Paraná vem apresentando uma tendência de aumento no volume pluviométrico, principalmente nos meses de verão e de primavera. No verão, o total de chuva teve um acréscimo de 17 a $37 \mathrm{~mm}$ por década, enquanto na primavera esta tendência foi de 16 a $42 \mathrm{~mm}$, sendo que algumas estaçôes hidrológicas apresentaram esta tendência significativa em ambos os períodos sazonais.

Damé et al. (2013) objetivaram identificar a presença ou ausência de tendência nas séries de precipitaçáo total anual da bacia hidrográfica da lagoa Mirim/RS, utilizando os dados de precipitação pertencentes a 14 estações climatológicas. Foram utilizados os testes não paramétricos de Mann-Kendall e Mann-Whitney, o teste " $\mathrm{t}$ " de Student para duas amostras de dados não pareados (paramétrico), bem como a técnica da média progressiva. Os autores concluíram que apenas a localidade de Pelotas apresentou alteração na tendência da série de precipitação no período de 1953 a 2007.

Diante do exposto, o presente trabalho teve como objetivo identificar a presença ou ausência de tendência nas séries de precipitação total mensal em 40 estaçôes pluviais, localizadas nas sete mesorregióes do estado do Rio Grande do Sul, totalizando para cada estaçáo, 42 anos (1961-2002).

\section{MATERIAL E MÉTODOS}

\subsection{Caracterização da região de estudo e clima}

O estado do Rio Grande do Sul ocupa uma área de aproximadamente $282.184 \mathrm{~km}^{2}$ e localiza-se entre as latitudes $27^{\circ} 05^{\prime} \mathrm{S}$ e $33^{\circ} 45^{\prime} \mathrm{S}$ e as longitudes $49^{\circ} 43^{\prime}$ O e 57³9' O. Segundo a classificação de Köppen, o estado se enquadra na zona fundamental temperada ou "C", isto é, com temperatura média do mês mais frio próxima a $3^{\circ} \mathrm{C}$, e no tipo fundamental "Cf”, clima temperado úmido, com chuvas distribuídas por todo ano. Como uma subdivisão do tipo fundamental "Cf", o estado costuma ser classificado em duas variedades específicas: clima subtropical ou virgiano, "Cfa", cuja temperatura do mês mais quente é superior a $22^{\circ} \mathrm{C}$, e a do mês mais frio oscila entre $3^{\circ} \mathrm{e} 18^{\circ} \mathrm{C}$ (ALVARES et al., 2013). A precipitação pluvial anual média é da ordem de $1.540 \mathrm{~mm}$, sendo superior a 1.500 mm na metade norte e inferior a este na metade sul (BERLATO et al., 2005).

\subsection{Levantamento dos dados}

Foram utilizadas 40 estaçôes pluviométricas distribuídas nas sete mesorregiôes do estado (IBGE, 2015) (Figura 1), obtidasa partirdo banco de dados da Agência Nacional de Águas (ANA, 2013), selecionando-as em função da existência de um período comum de dados e períodos sem falhas. $\mathrm{Na}$ Tabela 1 encontra-se a caracterização das estaçôes pluviais, quanto masoregiáo, número e nome da estação, latitude, longitude e altitude. 
Figura 1. Divisão do estado do Rio Grande do Sul em mesorregiões

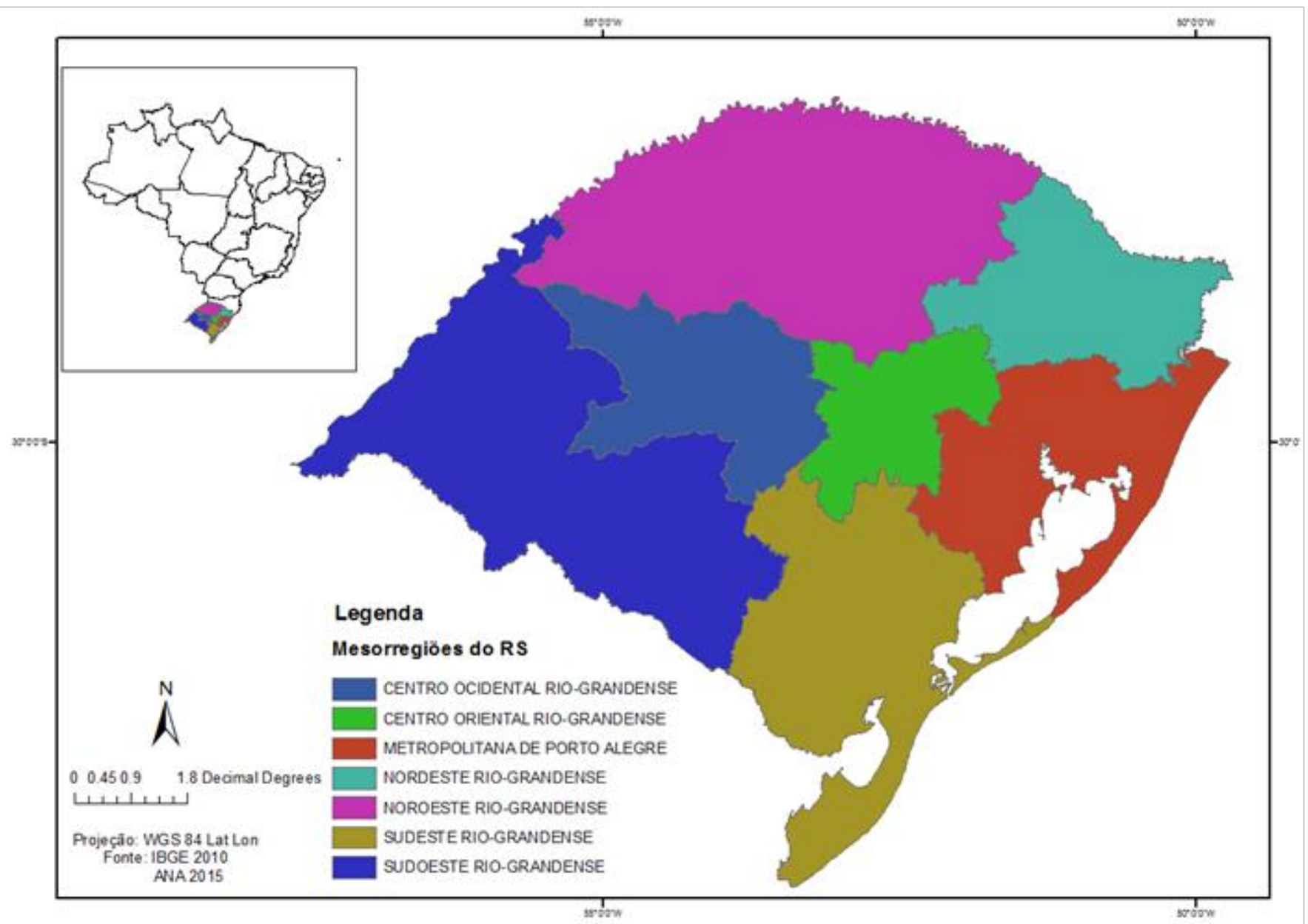

Tabeta 1. Caracterização das 40 estações de precipitação localizadas nas sete mesorregiões do estado do Rio Grande do Sul

\begin{tabular}{|c|c|c|c|c|c|}
\hline Mesorregião & Estação & Nome & Latitude & Longitude & Altitude (m) \\
\hline \multirow[t]{3}{*}{ Centro Ocidental } & 2953017 & Santa Maria & $29^{\circ} 43^{\prime} 27^{\prime \prime}$ & $53^{\circ} 43^{\prime} 12^{\prime \prime}$ & 95 \\
\hline & 2953011 & Júlio de Castilhos & $29^{\circ} 13^{\prime} 00^{\prime \prime}$ & $53^{\circ} 40^{\prime} 00^{\prime \prime}$ & 516 \\
\hline & 2954011 & Santiago & $29^{\circ} 11^{\prime} 00^{\prime \prime}$ & $54^{\circ} 52^{\prime} 00^{\prime \prime}$ & 426 \\
\hline \multirow[t]{3}{*}{ Centro Oriental } & 2955012 & Santa Cruz do Sul & $29^{\circ} 43^{\prime} 00^{\prime \prime}$ & $56^{\circ} 26^{\prime} 00^{\prime \prime}$ & 53 \\
\hline & 3052002 & Cachoeira do Sul & $30^{\circ} 02^{\prime} 00^{\prime \prime}$ & $52^{\circ} 53^{\prime} 00^{\prime \prime}$ & 73 \\
\hline & 2951030 & Taquari & $29^{\circ} 48^{\prime} 00^{\prime \prime}$ & $51^{\circ} 49^{\prime} 00^{\prime \prime}$ & 76 \\
\hline \multirow[t]{4}{*}{ Metropolitana } & 2950021 & Osório & $29^{\circ} 53^{\prime} 00^{\prime \prime}$ & $56^{\circ} 16^{\prime} 00^{\prime \prime}$ & 10 \\
\hline & 3051011 & Porto Alegre & $30^{\circ} 03^{\prime} 13^{\prime \prime}$ & $51^{\circ} 10^{\prime} 24^{\prime \prime}$ & 47 \\
\hline & 3051013 & Tapes & $30^{\circ} 40^{\prime} 00^{\prime \prime}$ & $51^{\circ} 24^{\prime} 00^{\prime \prime}$ & 5 \\
\hline & 2949002 & Torres & $29^{\circ} 20^{\prime} 41^{\prime \prime}$ & $49^{\circ} 43^{\prime} 41^{\prime \prime}$ & 31 \\
\hline \multirow[t]{7}{*}{ Nordeste } & 2951003 & Bento Gonçalves & $29^{\circ} 09^{\prime} 00^{\prime \prime}$ & $51^{\circ} 31^{\prime} 00^{\prime \prime}$ & 640 \\
\hline & 2851011 & Guaporé & $28^{\circ} 55^{\prime} 00^{\prime \prime}$ & $51^{\circ} 54^{\prime} 00^{\prime \prime}$ & 471 \\
\hline & 2951008 & Caxias do Sul & $29^{\circ} 11^{\prime} 39^{\prime \prime}$ & $51^{\circ} 11^{\prime 09}{ }^{\prime \prime}$ & 760 \\
\hline & 2850011 & Vacaria & $28^{\circ} 31^{\prime} 00^{\prime \prime}$ & $50^{\circ} 57^{\prime} 00^{\prime \prime}$ & 1050 \\
\hline & 2951013 & Farroupilha & $29^{\circ} 13^{\prime} 00^{\prime \prime}$ & $51^{\circ} 19^{\prime} 00^{\prime \prime}$ & 750 \\
\hline & 2851042 & Veranópolis & $28^{\circ} 56^{\prime} 14^{\prime \prime}$ & $51^{\circ} 33^{\prime} 11^{\prime \prime}$ & 705 \\
\hline & 2850001 & Bom Jesus & $28^{\circ} 40^{\prime} 07^{\prime \prime}$ & $50^{\circ} 26^{\prime} 31^{\prime \prime}$ & 1048 \\
\hline
\end{tabular}

Continua... 


\begin{tabular}{|c|c|c|c|c|c|}
\hline & 2851014 & Lagoa Vermelha & $28^{\circ} 13^{\prime} 19^{\prime \prime}$ & $51^{\circ} 30^{\prime} 45^{\prime \prime}$ & 842 \\
\hline & 2950032 & S. Francisco de Paula & $29^{\circ} 20^{\prime} 00^{\prime \prime}$ & $50^{\circ} 31^{\prime} 00^{\prime \prime}$ & 922 \\
\hline \multirow[t]{10}{*}{ Noroeste } & 2753005 & Palmeira das Missões & $27^{\circ} 53^{\prime} 00^{\prime \prime}$ & $53^{\circ} 26^{\prime} 00^{\prime \prime}$ & 634 \\
\hline & 2835005 & Cruz Alta & $28^{\circ} 37^{\prime} 28^{\prime \prime}$ & $53^{\circ} 36^{\prime} 12^{\prime \prime}$ & 432 \\
\hline & 2853025 & ljuí & $28^{\circ} 23^{\prime} 17^{\prime \prime}$ & $53^{\circ} 54^{\prime} 50^{\prime \prime}$ & 448 \\
\hline & 2754005 & Santa Rosa & $27^{\circ} 51^{\prime} 00^{\prime \prime}$ & $54^{\circ} 25^{\prime} 00^{\prime \prime}$ & 360 \\
\hline & 2852020 & Passo Fundo & $28^{\circ} 13^{\prime} 33^{\prime \prime}$ & $52^{\circ} 24^{\prime} 12^{\prime \prime}$ & 684 \\
\hline & 2852045 & Soledade & $28^{\circ} 50^{\prime} 00^{\prime \prime}$ & $52^{\circ} 26^{\prime} 00^{\prime \prime}$ & 720 \\
\hline & 2854007 & Santo Ângelo & $28^{\circ} 18^{\prime} 00^{\prime \prime}$ & $54^{\circ} 16^{\prime} 00^{\prime \prime}$ & 289 \\
\hline & 2751005 & Marcelino Ramos & $27^{\circ} 27^{\prime} 00^{\prime \prime}$ & $51^{\circ} 55^{\prime} 00^{\prime \prime}$ & 414 \\
\hline & 2854011 & São Luiz Gonzaga & $28^{\circ} 24^{\prime} 00^{\prime \prime}$ & $54^{\circ} 58^{\prime} 00^{\prime \prime}$ & 245 \\
\hline & 2753003 & Iraí & $27^{\circ} 11^{\prime} 20^{\prime \prime}$ & $53^{\circ} 15^{\prime} 12^{\prime \prime}$ & 247 \\
\hline \multirow[t]{5}{*}{ Sudeste } & 3053002 & Caçapava do Sul & $30^{\circ} 30^{\prime} 00^{\prime \prime}$ & $53^{\circ} 29^{\prime} 00^{\prime \prime}$ & 450 \\
\hline & 3152014 & Pelotas & $31^{\circ} 45^{\circ} 00^{\prime \prime}$ & $52^{\circ} 21^{\prime} 00^{\prime \prime}$ & 13 \\
\hline & 3252020 & Rio Grande & $32^{\circ} 04^{\prime} 44^{\prime \prime}$ & $52^{\circ} 10^{\prime} 00^{\prime \prime}$ & 3 \\
\hline & 3353007 & Sta Vitória do Palmar & $33^{\circ} 31^{\prime} 57^{\prime \prime}$ & $53^{\circ} 20^{\prime} 58^{\prime \prime}$ & 24 \\
\hline & 3052005 & Encruzilhada do Sul & $30^{\circ} 32^{\prime} 37^{\prime \prime}$ & $52^{\circ} 31^{\prime} 25^{\prime \prime}$ & 428 \\
\hline \multirow[t]{6}{*}{ Sudoeste } & 3053002 & Alegrete & $29^{\circ} 47^{\prime} 00^{\prime \prime}$ & $55^{\circ} 46^{\prime} 00^{\prime \prime}$ & 121 \\
\hline & 3055001 & S. do Livramento & $30^{\circ} 53^{\prime} 00^{\prime \prime}$ & $55^{\circ} 32^{\prime} 00^{\prime \prime}$ & 328 \\
\hline & 3054011 & São Gabriel & $30^{\circ} 20^{\prime} 00^{\prime \prime}$ & $54^{\circ} 19^{\prime} 00^{\prime \prime}$ & 124 \\
\hline & 3154001 & Bagé & $31^{\circ} 18^{\prime} 17^{\prime \prime}$ & $54^{\circ} 07^{\prime} 11^{\prime \prime}$ & 230 \\
\hline & 2957001 & Uruguaiana & $29^{\circ} 45^{\prime} 00^{\prime \prime}$ & $57^{\circ} 05^{\prime} 00^{\prime \prime}$ & 62 \\
\hline & 2856005 & São Borja & $28^{\circ} 39^{\prime} 44^{\prime \prime}$ & $56^{\circ} 00^{\prime} 44^{\prime \prime}$ & 83 \\
\hline
\end{tabular}

\subsection{Análise de tendência}

Após a identificação visual do comportamento de tendência foram aplicados os testes não-paramétricos de Mann-Kendall (existência de tendência monotônica) e de Mann-Whitney (pontos de mudança) para aceitar ou rejeitar a hipótese de nulidade, $\mathrm{H}_{0}$, que refere-se a não existência de alteração de tendência na população de onde foi retirada a amostra dos dados, enquanto que a hipótese alternativa, $\mathrm{H}_{1}$, refere-se a existência de tendência nos registros.

Para testar as hipóteses de tendência de longo prazo e a dos passos de mudança, as metodologias empregadas fundamentaram-se em: (a) verificação da existência ou não de tendência monotônica e; (b) detecção dos pontos de mudança, caso estas ocorram.

A identificação do período em que as séries deveriam ser subdivididas fundamentou-se na observação visual do comportamento da média móvel com retardo $(\mathrm{k})$ igual a 10 , em relação a média dos valores totais anuais precipitados.

Para a verificação da existência ou não de tendência monotônica foi utilizado o teste não paramétrico de Mann-Kendall (XU et al., 2003), sendo a estatística do teste dada pela Eq. 1:

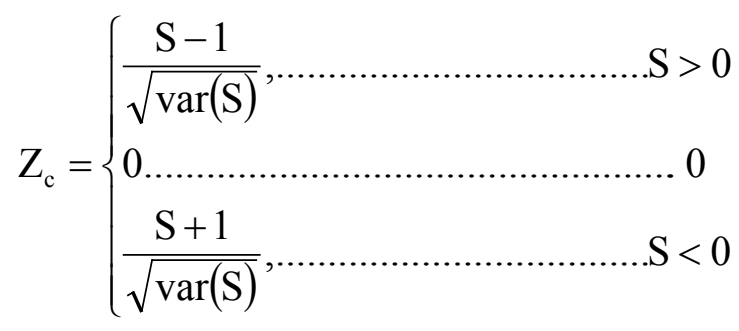

em que,

Zc - estatística do teste;

S - estatística do teste que segue a distribuição normal, cuja média E (.) e variância var (.), representados matematicamente pelas Eqs. 3, 4 e 5, respectivamente.

$\mathrm{E}(\mathrm{S})=0$

$\mathrm{S}=\sum_{\mathrm{i}=1}^{\mathrm{n}-1} \sum_{\mathrm{k}=\mathrm{i}+1}^{\mathrm{n}} \operatorname{sgn}\left(\mathrm{x}_{\mathrm{k}}-\mathrm{x}_{\mathrm{i}}\right)$

$\operatorname{var}(\mathrm{S})=\frac{\mathrm{n}(\mathrm{n}-1)(2 \mathrm{n}+5)}{8}$

em que, 
$\mathrm{x}_{\mathrm{k}}$ e $\mathrm{x}_{\mathrm{i}}$ - valores contidos na amostra com ordem $\mathrm{k} \mathrm{e}$ i, respectivamente;

sgn $(\theta)$ - igual a 1,0 , e -1 , se $\theta\left(\mathrm{x}_{\mathrm{k}}\right.$ e $\left.\mathrm{x}_{\mathrm{i}}\right)$ for maior, igual ou menor do que zero, respectivamente;

$\mathrm{n}$ - tamanho da amostra.

A hipótese de nulidade é aceita se $\left|Z_{c}\right| \leq Z_{1-\alpha / 2}$, em que $Z_{1-\alpha / 2}$, é o quartil da distribuição normal padrão correspondente ao nível $\alpha(0,05)$ de probabilidade, usado para a aplicação do teste.

Para todas as séries de precipitação consideradas independentes foram construídos gráficos com o propósito de detectar visualmente o ponto em que houve a intersecção da variável em relação à média móvel com lag 10, correspondente a localização do ponto aproximado de mudança de tendência. $\mathrm{Na}$ aplicação do teste não paramétrico de Mann-Whitney (CHEN et al., 2007) deve-se seccionar a série de dados $\mathrm{X}=\left(\mathrm{x}_{1}, \mathrm{x}_{2}, \mathrm{x}_{3}, \ldots ., \mathrm{x}_{\mathrm{n}-1}, \mathrm{x}_{\mathrm{n}}\right)$, de tal forma que $\mathrm{Y}=\left(\mathrm{x}_{1}, \mathrm{x}_{2}\right.$, $\left.\mathrm{x}_{3}, \ldots ., \mathrm{x}_{\mathrm{n} 1}\right)$ e $\mathrm{Z}=\left(\mathrm{x}_{\mathrm{n}+1}, \mathrm{x}_{\mathrm{n}+2}, \mathrm{x}_{\mathrm{n}+3}, \ldots . ., \mathrm{x}_{\mathrm{n} 1+\mathrm{n} 2}\right)$. A estatística do teste é dada pela Eq. 5. A hipótese de nulidade, $H_{0}$, é aceita se $\left|Z_{c}\right| \leq Z_{1-\alpha / 2}$, onde $Z_{1-\alpha / 2}$ é o quartil da distribuição normal padrão correspondente ao nível $\alpha(0,05)$ de probabilidade, usado para a aplicação do teste. Todas as análises de tendência, incluindo a de independência foram realizadas utilizando planilhas Excel.

$\mathrm{Z}_{\mathrm{c}}=\frac{\sum_{\mathrm{t}=1}^{\mathrm{n}_{1}} \mathrm{r}\left(\mathrm{x}_{\mathrm{t}}\right)-\mathrm{n}_{1}\left(\mathrm{n}_{1}+\mathrm{n}_{2}+1\right) / 2}{\left[\mathrm{n}_{1} \mathrm{n}_{2}\left(\mathrm{n}_{1}+\mathrm{n}_{2}+1\right) / \mathcal{L}\right]^{1 / 2}}$

em que,

$r\left(x_{t}\right)$ - ordem das observaçóes;

$\mathrm{n}_{1}$ e $\mathrm{n}_{2}$ - número de elementos das sub-amostras $\mathrm{X}$ e Y, respectivamente.

\section{RESULTADOS E DISCUSSÃO}

\subsection{Estatísticas descritivas das 40 estações analisadas}

$\mathrm{Na}$ Tabela 2 são apresentados os valores das estatísticas descritivas, desvio padrão, coeficiente de variação, coeficiente de assimetria, máximo e mínimo de todas as séries de precipitação analisadas, para as sete mesorregióes do Estado do Rio Grande do Sul, no período de 1961 a 2002. A amplitude dos valores do coeficiente de variação foram de 0,38 a 0,70 , para as séries de precipitação total mensal, para as localidades de Soledade (mesorregiáo Noroeste) e Uruguaiana (mesorregião Sudoeste), respectivamente, o que indica elevada dispersão dos dados. Gomes et al. (2015) analisaram os dados médios mensais de precipitação de 102 estaçôes meteorológicas distribuídas espacialmente no estado da Paraíba, no período de 1962 a 2001 e encontraram valores de coeficiente de variação entre 0,31 e 1,34 , que os fizeram concluir que as chuvas mensais possuem alta dispersão, visto que segundo Oliveira (1999), valores de coeficiente de variaçáo maiores do que 0,20 indicam elevada dispersáo dos dados. Comparando-se os valores de coeficiente de assimetria observa-se que somente a estação de Tapes (mesorregião Metropolitana) é simétrica $(\mathrm{Ca}=1,00)$. Em contrapartida, para as demais localidades, todos os dados exibem assimetria positiva, cujos valores de Ca variam de 0,63 (Soledade) a 2,89 (São Francisco de Paula), caracterizando a não ocorrência da normalidade de distribuição de frequência nos dados $(\mathrm{Ca}=0)(\mathrm{QUADROS}$ et al., 2011).

\subsection{Caracterização do regime pluvial das sete mesorregiões}

Observa-se na Figura 2 que as médias dos valores de precipitação das estaçôes, nas diferentes mesorregióes não apresentaram uma característica de regime pluviométrico bem definido, ou seja, não há como definir com clareza as estaçóes chuvosas e secas, evidenciando que na região sul, a distribuição da precipitação média mensal mostra-se regular, oscilando entre 115,28 e $153,36 \mathrm{~mm}$, para os meses de novembro e setembro, respectivamente.

Blain et al. (2009) avaliaram a variabilidade amostral dos parâmetros da distribuição Gama relativos a séries mensais de precipitação pluvial de apenas duas regióes (Campinas-SP e Pelotas-RS), para um maior entendimento do tema variaçóes/mudanças climáticas. Os autores verificaram que ao contrário do observado na região de Campinas-SP, que possui uma estação seca definida, de maio a agosto, na região de Pelotas-RS, as chuvas são distribuídas de forma semelhante ao longo do ano. 
Tabela 2. Estatísticas descritivas dos valores mensais de precipitação das 40 estações analisadas, nas sete mesorregiões do estado do Rio Grande do Sul

\begin{tabular}{|c|c|c|c|c|c|c|}
\hline Mesorregião & Estação & $\mathrm{DP}(\mathrm{mm})$ & $\mathrm{CV}$ & $\mathrm{Ca}$ & Máx.(mm) & Mín. (mm) \\
\hline \multirow[t]{3}{*}{ Centro Ocidental } & 2953017 & 83,7 & 0,58 & 0,84 & 476,8 & 0,2 \\
\hline & 2953011 & 76,0 & 0,57 & 0,85 & 489,0 & 4,0 \\
\hline & 2954011 & 69,7 & 0,53 & 1,11 & 497,2 & 7,5 \\
\hline \multirow[t]{3}{*}{ Centro Oriental } & 2955012 & 61,3 & 0,49 & 1,49 & 556,9 & 3,1 \\
\hline & 3052002 & 84,1 & 0,61 & 1,73 & 745,3 & 2,9 \\
\hline & 2951030 & 68,6 & 0,53 & 0,65 & 389,3 & 0,7 \\
\hline \multirow[t]{4}{*}{ Metropolitana } & 2950021 & 80,9 & 0,59 & 1,11 & 474,3 & 3,5 \\
\hline & 3051011 & 63,8 & 0,55 & 0,77 & 365,6 & 0,5 \\
\hline & 3051013 & 60,1 & 0,54 & 1,00 & 393,3 & 1,4 \\
\hline & 2949002 & 73,2 & 0,60 & 1,12 & 416,8 & 4,7 \\
\hline \multirow[t]{9}{*}{ Nordeste } & 2951003 & 74,3 & 0,52 & 0,71 & 461,5 & 8,3 \\
\hline & 2851011 & 78,0 & 0,52 & 1,03 & 565,0 & 2,9 \\
\hline & 2951008 & 75,5 & 0,50 & 0,77 & 449,7 & 8,2 \\
\hline & 2850011 & 66,4 & 0,52 & 1,16 & 532,9 & 0,0 \\
\hline & 2951013 & 72,3 & 0,51 & 0,82 & 438,6 & 5,5 \\
\hline & 2851042 & 75,6 & 0,55 & 1,01 & 446,8 & 5,6 \\
\hline & 2850001 & 79,2 & 0,55 & 1,39 & 607,1 & 9,6 \\
\hline & 2851014 & 73,6 & 0,51 & 0,94 & 483,0 & 1,6 \\
\hline & 2950032 & 84,1 & 0,44 & 2,89 & 915,0 & 36,4 \\
\hline \multirow[t]{10}{*}{ Noroeste } & 2753005 & 77,8 & 0,51 & 0,88 & 504,0 & 7,2 \\
\hline & 2835005 & 89,1 & 0,59 & 1,14 & 617,6 & 10,2 \\
\hline & 2853025 & 83,8 & 0,58 & 1,09 & 523,9 & 6,0 \\
\hline & 2754005 & 85,9 & 0,59 & 1,16 & 615,7 & 6,6 \\
\hline & 2852020 & 86,8 & 0,57 & 1,01 & 550,4 & 8,0 \\
\hline & 2852045 & 62,3 & 0,38 & 0,63 & 425,1 & 12,3 \\
\hline & 2854007 & 69,7 & 0,53 & 1,11 & 497,2 & 7,5 \\
\hline & 2751005 & 61,7 & 0,42 & 1,27 & 494,1 & 11,5 \\
\hline & 2854011 & 91,9 & 0,58 & 0,86 & 538,3 & 7,1 \\
\hline & 2753003 & 80,9 & 0,53 & 0,85 & 518,3 & 6,9 \\
\hline \multirow[t]{5}{*}{ Sudeste } & 3053002 & 76,3 & 0,51 & 1,41 & 608,7 & 17,5 \\
\hline & 3152014 & 71,9 & 0,62 & 1,31 & 510,0 & 3,7 \\
\hline & 3252020 & 67,6 & 0,65 & 1,62 & 521,8 & 3,6 \\
\hline & 3353007 & 67,7 & 0,65 & 1,57 & 473,3 & 0,3 \\
\hline & 3052005 & 79,2 & 0,61 & 1,30 & 556,4 & 1,1 \\
\hline \multirow[t]{6}{*}{ Sudoeste } & 3053002 & 80,1 & 0,60 & 1,12 & 517,1 & 2,7 \\
\hline & 3055001 & 75,5 & 0,61 & 1,31 & 430,4 & 4,8 \\
\hline & 3054011 & 72,7 & 0,60 & 1,12 & 452,6 & 0,7 \\
\hline & 3154001 & 84,0 & 0,66 & 1,37 & 532,0 & 1,1 \\
\hline & 2957001 & 91,3 & 0,70 & 1,15 & 528,1 & 1,5 \\
\hline & 2856005 & 84,2 & 0,62 & 1,24 & 529,2 & 4,0 \\
\hline
\end{tabular}




\subsection{Análise da independência}

$\mathrm{Na}$ Tabela 4 são apresentados os valores do coeficiente de autocorrelação de ordem $\mathrm{k}=1$, que mostram que das 40 séries temporais de precipitaçáo analisadas, 6 são consideradas ruído branco, ou seja, cada valor da série tem média zero, variância constante e não apresenta correlação serial, visto que todos os coeficientes de autocorrelaçáo estáo dentro do intervalo de confiança, LI $=-0,090$ e $\mathrm{LS}=0,090$ (LUCAS et al., 2009). Como os valores das séries temporais de precipitação analisadas não apresentaram autocorrelação serial significativa, os 6 municípios (Vacaria, Ijuí, Santa Rosa, Iraí, Pelotas e Santana do Livramento) são considerados independentes e os testes estatísticos de tendência podem ser aplicados (ULIANA et al., 2014).

Figura 2. Precipitação média mensal das estações que compõem as diferentes mesorregiões do estado do Rio Grande do Sul, no período de 1961-2002
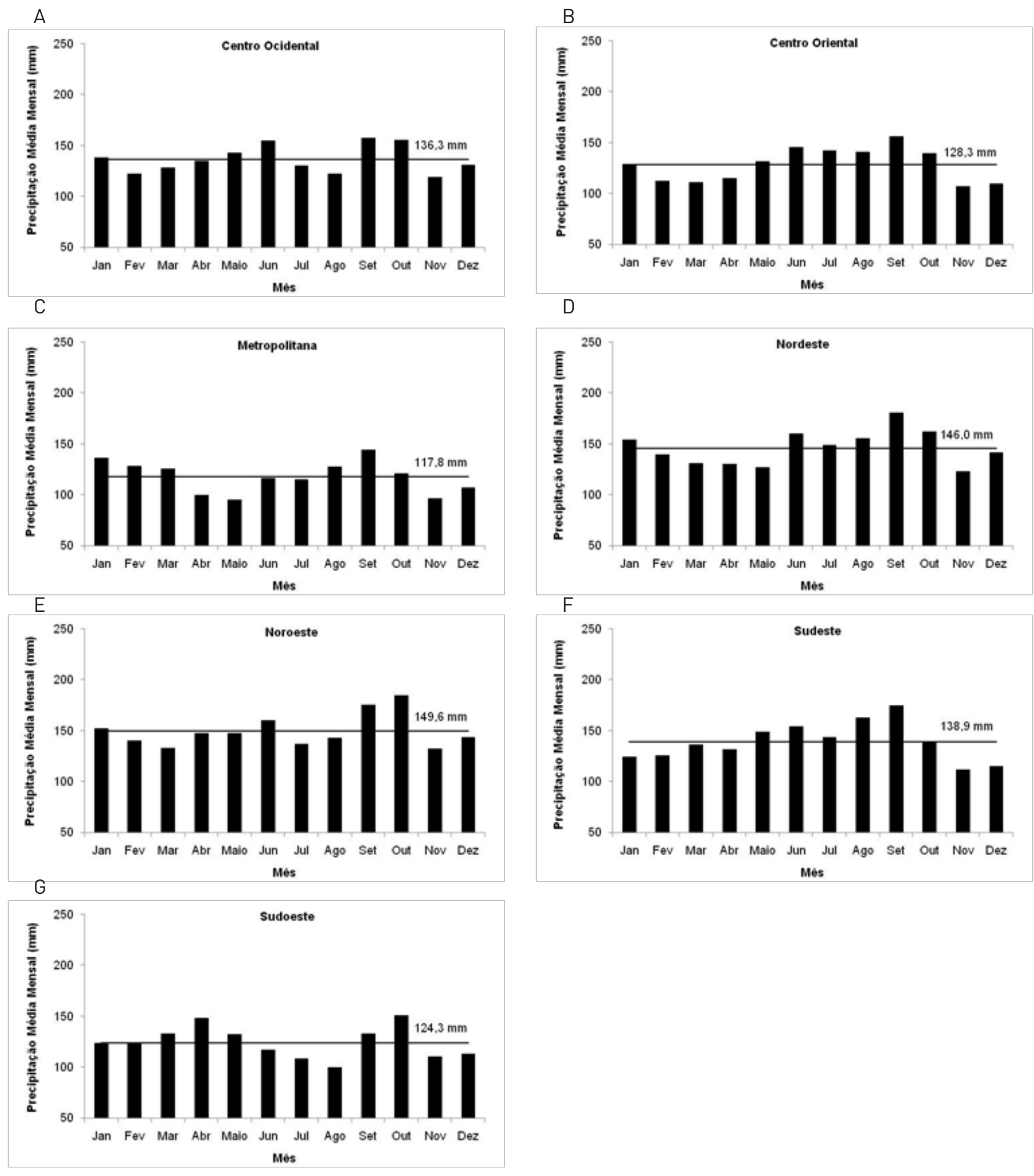
Tabela 3. Valores do coeficiente de autocorrelação de ordem $\mathrm{k}=1$, para as séries de precipitação total mensal que compõem as mesorregiões do estado do RS

\begin{tabular}{|c|c|c|c|}
\hline Mesorregião & Estação & Nome & $r_{k}^{*}$ \\
\hline \multirow[t]{3}{*}{ Centro Ocidental } & 2953017 & Santa Maria & 0,123 \\
\hline & 2953011 & Júlio de Castilhos & 0,164 \\
\hline & 2954011 & Santiago & 0,188 \\
\hline \multirow[t]{3}{*}{ Centro Oriental } & 2955012 & Santa Cruz do Sul & 0,155 \\
\hline & 3052002 & Cachoeira do Sul & 0,299 \\
\hline & 2951030 & Taquari & 0,139 \\
\hline \multirow[t]{4}{*}{ Metropolitana } & 2950021 & Osório & 0,131 \\
\hline & 3051011 & Porto Alegre & 0,142 \\
\hline & 3051013 & Tapes & 0,119 \\
\hline & 2949002 & Torres & 0,159 \\
\hline \multirow[t]{9}{*}{ Nordeste } & 2951003 & Bento Gonçalves & 0,142 \\
\hline & 2851011 & Guaporé & 0,186 \\
\hline & 2951008 & Caxias do Sul & 0,150 \\
\hline & 2850011 & Vacaria & 0,085 \\
\hline & 2951013 & Farroupilha & 0,192 \\
\hline & 2851042 & Veranópolis & 0,141 \\
\hline & 2850001 & Bom Jesus & 0,165 \\
\hline & 2851014 & Lagoa Vermelha & 0,160 \\
\hline & 2950032 & $\begin{array}{l}\text { S. Francisco de } \\
\text { Paula }\end{array}$ & 0,128 \\
\hline \multirow[t]{10}{*}{ Noroeste } & 2753005 & $\begin{array}{l}\text { Palmeira das } \\
\text { Missões }\end{array}$ & 0,117 \\
\hline & 2835005 & Cruz Alta & 0,151 \\
\hline & 2853025 & ljuí & 0,076 \\
\hline & 2754005 & Santa Rosa & 0,078 \\
\hline & 2852020 & Passo Fundo & 0,115 \\
\hline & 2852045 & Soledade & 0,096 \\
\hline & 2854007 & Santo Ângelo & 0,115 \\
\hline & 2751005 & Marcelino Ramos & 0,132 \\
\hline & 2854011 & São Luiz Gonzaga & 0,104 \\
\hline & 2753003 & Iraí & 0,034 \\
\hline \multirow[t]{5}{*}{ Sudeste } & 3053002 & Caçapava do Sul & 0,199 \\
\hline & 3152014 & Pelotas & 0,085 \\
\hline & 3252020 & Rio Grande & 0,113 \\
\hline & 3353007 & $\begin{array}{l}\text { Sta Vitória do } \\
\text { Palmar }\end{array}$ & 0,120 \\
\hline & 3052005 & Encruzilhada do Sul & 0,180 \\
\hline \multirow[t]{6}{*}{ Sudoeste } & 3053002 & Alegrete & 0,151 \\
\hline & 3055001 & S. do Livramento & 0,084 \\
\hline & 3054011 & São Gabriel & 0,106 \\
\hline & 3154001 & Bagé & 0,121 \\
\hline & 2957001 & Uruguaiana & 0,144 \\
\hline & 2856005 & São Borja & 0,162 \\
\hline
\end{tabular}

\subsection{Análise de tendência}

Quando foi aplicado o teste de Mann-Kendall (Tabela 4), que considera o período total da série (1961 a 2002) para as 6 estaçóes consideradas independentes, não houve evidência de alteração de tendência, uma vez que $Z_{\text {calc }}$ foi inferior ao valor crítico da estatística $(1,96)$ para o nível de probabilidade de 5\%. Dentre os não-paramétricos, o teste de Mann-Kendall considera que na hipótese de estabilidade de uma série temporal, a sucessão de valores ocorre de forma independente, e a distribuição de probabilidade não deve ser alterada ao longo do tempo (série aleatória simples). O teste é considerado significativo quando a estatística do teste está dentro do intervalo de confiança, ou seja, assim a hipótese de nulidade $\left(\mathrm{H}_{0}\right)$ deve ser rejeitada sempre que o valor da estatística estiver fora do intervalo de confiança, para um nível $\alpha$ de probabilidade (SILVA et al., 2011; BLAIN, 2011).

Tabela5.Valores da estatística de Mann-Kendall para as 6 estações analisadas

\begin{tabular}{llll} 
Estação & Nome & $\mathrm{Z}_{\text {calc }}$ & $\mathrm{H}_{0}(\mathrm{Z})$ \\
\hline 2853025 & ljuí & 0,001 & $\mathrm{~A}$ \\
2754005 & Santa Rosa & $-0,002$ & $\mathrm{~A}$ \\
2753003 & Iraí & 0,000 & $\mathrm{~A}$ \\
3152014 & Pelotas & 0,002 & $\mathrm{~A}$ \\
3055001 & S. do Livramento & $-0,004$ & $\mathrm{~A}$ \\
2850011 & Vacaria & 0,002 & $\mathrm{~A}$ \\
\hline & $\mathrm{Z}_{\text {Tab }}=1,96$ para $5 \%$ de probabilidade
\end{tabular}

Os resultados gráficos do comportamento das séries de precipitação total mensal, com suas respectivas médias e médias móveis de lag 10, para as seis localidades consideradas independentes, no período de 1961 a 2002 estão apresentados na Figura 3. Além da precipitação, as linhas horizontais indicam as médias, o ajuste linear, bem como o comportamento das médias móveis de lag 10. 


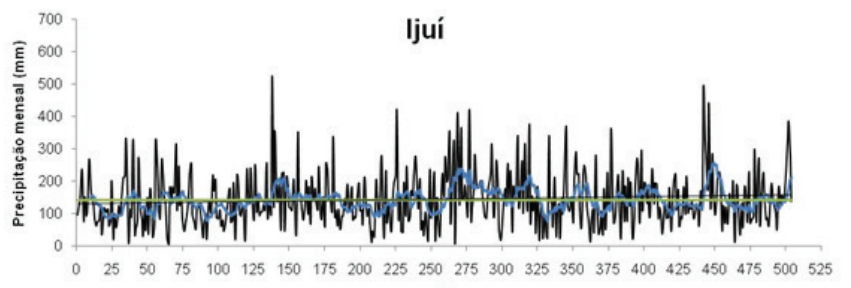
Mes
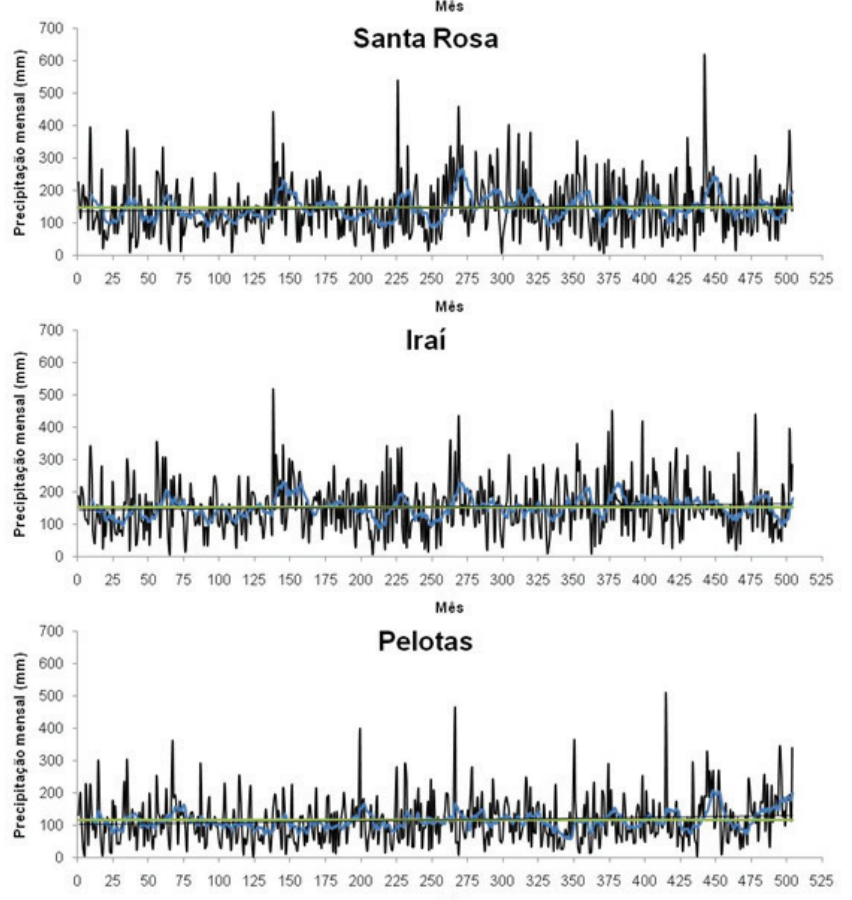

Mès

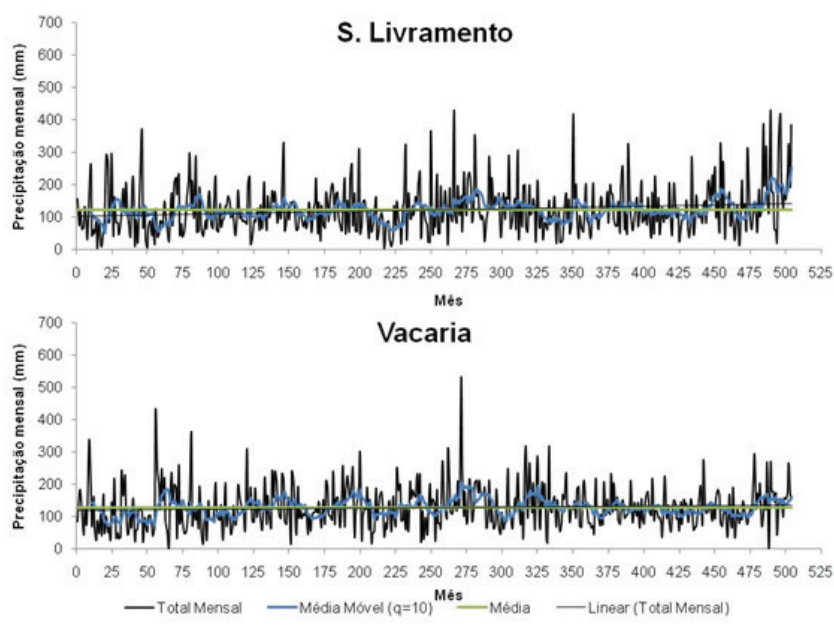

Figura 3. Representação do comportamento das séries de precipitação total mensal das seis localidades, com suas respectivas médias, médias móveis de lag 10 e ajuste linear

A Tabela 5 mostra os resultados do teste de MannWhitney, bem como a sua interpretação para as seis localidades analisadas. Observa-se que apenas para a localidade de Pelotas, cujo valor de Zcalc $(2,43)$ é maior do que o Ztab $(1,96)$ apresenta alteração de tendência, rejeitando-se a hipótese de nulidade $\left(\mathrm{H}_{0}\right)$. Os resultados mostraram que a alteração de tendência ocorreu a partir de janeiro de 1988, com aumento nos valores médios, em que no primeiro período (janeiro de 1961 a dezembro de 1987 - 324 valores) o valor médio foi de 112,0 mm e no segundo período (janeiro de 1988 a dezembro de 2002 - 180 valores), a média foi de $121,0 \mathrm{~mm}$. Com relação às demais, não foi detectada alteração em seu comportamento. Fontolan et al. (2012) identificaram tendências anuais da temperatura e precipitação pluvial de cinco cidades que se situam ao longo do rio Tietê/SP, utilizando análises de regressão linear e os testes não paramétricos de Run, Mann-Kendall e Pettitt, que é um procedimento que utiliza uma versão do teste de Mann-Whitney. Os autores encontraram que apenas a cidade de Araraquara apresentou tendência de decrescimento da precipitação pluvial, confirmado no ano 1983. No caso da localidade em análise é importante ressaltar que o teste de Mann-Whitney, que tem como metodologia particionar a série, detectou alteração na tendência, comparativamente ao teste de Mann-Kendall que utiliza a série completa (504 valores).

Tabela 6. Resultados do teste de Mann-Whitney e interpretação para as seis localidades do estado do Rio Grande do Sul

\begin{tabular}{lccl} 
Localidade & Zcalc & Ztab* & Interpretação \\
\hline ljuí & $-1,35$ & $\pm 1,96$ & Aceita $\mathrm{H}_{0}$ \\
Santa Rosa & $-1,11$ & $\pm 1,96$ & Aceita $\mathrm{H}_{0}$ \\
Iraí & $-0,48$ & $\pm 1,96$ & Aceita $\mathrm{H}_{0}$ \\
Pelotas & 2,43 & $\pm 1,96$ & Rejeita $\mathrm{H}_{0}$ \\
Santana do Livramento & $-1,63$ & $\pm 1,96$ & Aceita $\mathrm{H}_{0}$ \\
Vacaria & 1,04 & $\pm 1,96$ & Aceita $\mathrm{H}_{0}$ \\
\hline \multicolumn{4}{c}{}
\end{tabular}

\section{CONCLUSÕES}

Das estaçóes consideradas independentes, apenas na localidade de Pelotas, a aplicação do teste de Mann-Whitney, que considerou a subdivisão da série (janeiro de 1961 a dezembro de 1987 e de janeiro de 1988 a dezembro de 2002) detectou alteração de tendência na série mensal total de precipitação. $\mathrm{Na}$ referida localidade, houve incremento nos valores totais mensais de precipitação para o período de 1961 a 2002.

\section{BIBLIOGRAFIA}

AGÊNCIA NACIONAL DE ÁGUAS (ANA). Sistema de informaçôes hidrológicas (HidroWeb). Disponível em: < http:// 
hidroweb.ana.gov.br/>. Acesso em 26 abr. 2013.

ALCÂNTARA, C.R.; FERREIRA, D.V.R.; SILVA, G.J.F; ALMEIDA, H.A. Aquecimento Global ou Variabilidade Climática Natural na Microrregiáo de Juazeiro - BA? Revista Brasileira de Geografia Física, v.03, p.572-585, 2012.

ALVARES, C.A.; STAPE, J.L.; SENTELHAS, P.C.; GONÇALVES, J.L.M.; SPAROVEK, G. Köppen's climate classification map for Brazil. Meteorologisch e Zeitschrift, p.113, 2013.

BERLATO, M. A.; FARENZEN, H; FONTANA, D. Associaçáo entre El Nińo Oscilaçáo Sul e a produtividade do milho no estado do Rio Grande do Sul. Pesquisa Agropecuária Brasileira, v.40, p.423-432, 2005.

BLAIN, G.C. Totais decendiais de precipitaçáo pluvial em Campinas, SP: persistência temporal, periodicidades e tendências climáticas. Ciência Rural, v.41, p.789-795, 2011.

BLAIN, G.C.; PICOLI, M.C.A.; LULU, J. Análises estatísticas das tendências de elevaçáo nas séries anuais de temperatura mínima do ar no Estado de Sáo Paulo. Bragantia, v.68, p.807815, 2009.

CHEN, Y.N.; LI, W.H.; XU, C.C.; HAO, X.M. Effects of climate change on water resources in Tarim River Basin, Northwest China. Journal of Environmental Sciences, v.19, p.488-493, 2007.

DAMÉ, R.C.F.; TEIXEIRA, C.F.A.; BACELAR, L.C.S.; WINKLER, A.S.; SANTOS, J.P. Monotonic trend and change points in southern Brazil precipitation. Revista Engenharia Agrícola, v.33, p.258-268, 2013.

FONTOLAN, M.R.; FERREIRA, D.H.L.; PENEREIRO, J.C. Comportamento climático ao longo do rio Tietê: aplicaçóes envolvendo testes estatísticos. Revista Geonorte, v.1, p.466475, 2012.

GOMES, O.M.; SANTOS, C.A.C.; SOUZA, S.S.; PAIVA, W.; OLINDA, R.A. Análise comparativa da precipitaçáo do estado da Paraíba utilizando modelos de regressáo polinomial. Revista Brasileira de Meteorologia, v.30, p.47-58, 2015.

INSTITUTO BRASILEIRO DE GEOGRAFIA E ESTATÍSTICA (IBGE). Mesorregióes geográficas. Capturado em 30 jan. 2015. Online.Disponívelemhttp://mapas.fee.tche.br/ wp-content/uploads/2011/11/Mesorregioes.pdf

LUCAS, E.W.M.; SOUSA, F.A.S.; SILVA, F.D.S.; LUCIO, P.S.

Modelagem hidrológica determinística e estocástica aplicada à regiáo hidrográfica do Xingu - Pará. Revista Brasileira de
Meteorologia, v.24, p.308-322, 2009.

MINUZZI, R.B.; CARAMORI, P.H. Variabilidade climática sazonal e anual da chuva e veranicos no Estado do Paraná. Revista Ceres, v.58, p.593-602, 2011.

OLIVEIRA, F.E.M. Estatística e Probabilidade. 2. ed. São Paulo: Atlas, ISBN 85-224-2103-X, 221 p. 1999.

QUADROS, L.E.; QUEIROZ, M.M.F.; VILAS BOAS, M.A. Distribuiçáo de frequência e temporal de chuvas intensas. Acta ScientiarumAgronomy, v.33, p.401-410, 2011.

RIBEIRO, R.E.P.; ÁVILA, P.L.R.; BRITO, J.I.B.; SANTOS, E.G.; SOUSA, L.F. Análise da tendência climática nas séries temporais de temperatura e precipitação de Tucuruí-Pará. Revista Brasileira de Geografia Física, v.07, p.798-807, 2014.

SANTOS, C.O.; SCALON, J.D.; OZAKI, V.A. A distribuição normal-assimétrica como modelo para produtividade de milho aplicada ao seguro agrícola. Revista de Economia e Sociologia Rural, v.52, p.725-742, 2014.

SILVA, F.D.S.; FERREIRA, D.B.; SARMANHO, G.F.; SANTOS, L.S.F.C.; FORTES, L.T.G.; PARENTE, E.G.P. Tendência de alteraçóes climáticas da precipitação observadas no Brasil de 1961 a 2008 utilizando dados gradeados. In: XII Congresso Brasileiro de Meteorologia, 2010, Belém. Anais... Belém: SBMET, 2010. CD Rom.

SILVA, V.P.R.; PEREIRA, E.R.R.; AZEVEDO, P.V.; SOUSA, F.A.S.; SOUSA, I.F. Análise da pluviometria e dias chuvosos na regiáo Nordeste do Brasil. Revista Brasileira de Engenharia Agrícola e Ambiental. v.15, p.131-138, 2011.

TEIXEIRA, C.F.A.; DAMÉ, R.C.F.; ROSSKOFF, J.L. Intensityduration-frequency ratios obtained from an nual records and partial duration records in the locality of Pelotas -RS, Brazil. Revista Engenharia Agrícola, v.31, p.687-694, 2011.

ULIANA, E.M.; SILVA, D.D.; ULIANA, E.M.; RODRIGUES, B.S.; CORRÊDO, L.P. Análise de tendência em séries históricas de vazáo e precipitaçáo: uso de teste estatístico não paramétrico. Revista Ambiente \& Água, v.10, p.82-88, 2015.

VENKATARAMANA, S.V.; INIYANB, S.; RANKO, G. A review of climate change, mitigation and adaptation. Renewable and Sustainable Energy Reviews, v.16, p.878- 897, 2012.

XU, Z.X.; TAKEUCHIA, K.; ISHIDAIRAA, H. Monotonic trend and step changes in Japanese precipitation. JournalofHydrology, v.279, p.144-150, 2003. 